\title{
Research on Social Organization Employee's Job Satisfaction in China Based on Multi-level Factor Analysis Method
}

\author{
Li Jian \\ School of management, MinZu University of China, Beijing, P.R. China 100081 \\ Email: bichenfei@163.com
}

\begin{abstract}
Human resource is the core resources of social organization, and social organization employee's job satisfaction is also the key research topic in charity research. On the basis of the national survey, this paper draws factor analysis method to evaluate employee's job satisfaction of 438 social organizations in China, and takes four principal components as measurement indicators of the criterion level through analytic hierarchy process, the ten domains of social organization as measurement indicators of the program level. The finding shows: (1) Social organization employee's job satisfaction scores low in China. (2) Employee recognition makes up the most important weight of social organization employee's job satisfaction. (3) The difference among the fields of social organization employee's job satisfaction is huge.
\end{abstract}

Keywords: Social Organization, Job Satisfaction, Multi-level Factor Analysis

\section{Introduction}

Social organization is a special organization with Chinese characteristic, which is similar with NGO, NPO and CSO. Social organizations are compose of foundation, non-governmental non-commercial enterprise and association, which are usually divided into ten domains, including education, health, culture, technology, sports, labor, civil affairs, social medium service, legal service and others. At present, a dilemma of social organization is the growing labour shortage, exacerbated by an increasing demand for human resource, fewer employees starting and more employees leaving the profession for the other sectors. According to the Ministry of Civil Affairs of the People's Republic of China, there were 440 thousand social organizations and 6.18 million employees, which accounts for only $0.5 \%$ of the population in 2010; the number of social organizations increased to 500 thousand in 2012, while the total employees decreased to 6.13 million, which accounted for only $0.48 \%$ of the population. The phenomenon draws increasing research attention to the motives, reward preferences and job satisfaction of staffs in social organization. A better understanding of these issues and of the facets affecting employee's intention to remain employed should benefit their retention. Unlike for-profit organization and public sector, however, research on social organization employee's job satisfaction remains scant. Job satisfaction is one of the strongest predictors of intent to stay and retention, and there is also some evidence that the quality of service is related to providers' job satisfaction ${ }^{[1]}$. A better understanding of social organization employee's job satisfaction can therefore benefit the sector significantly ${ }^{[2]}$.

\section{Literature Review}

Hale (1986) suggests that there is a lack of consensus as to what staff satisfaction is yet differences in definition may be more apparent than real ${ }^{[3]}$. For example, the definition given by Pilkington \& Wood (1986) ${ }^{[4]}$, like that of Blegen \& Mueller (1987), is that staff satisfaction is the extent of positive affective orientation to the job ${ }^{[5]}$. Bush (1988) gives a more elaborate 
definition of job satisfaction as, the perception that one's job fulfills or allows the fulfillment of one's important job values, providing and to the degree that those values are congruent with one's needs ${ }^{[6]}$.

A few researchers studied the employees' satisfaction in social organization context, while the findings are ambiguous. Salamon (1995) indicated employees in nonprofit organization worked in a uncertain conditions and faced serious pressure of financial, political and managerial, so the social organization employees' job satisfaction used to be lower than the other sector ${ }^{[7]}$. While another studies didn't think so, a cross-national investigation about social workers found they were alive, happy and prospering, and had a high job satisfaction and low departure will ${ }^{[8]}$. Hamarta (2009) also pointed out social problem solving is a self-esteem behavior, which can enhance the employees' job satisfaction ${ }^{[9]}$.

With regard to method, both factor analysis and analytic hierarchy process have been used to identify the underlying dimensions of employees' satisfaction, but the reasons for the choice of analysis and details of the actual analysis are not always given. These two approaches appear similar in that both are used to achieve parsimony, to reduce a large number of variables to a smaller number of underlying dimensions of factors. Yet factor analysis and analytic hierarchy process differ in important respects. Factor analysis, of which there are several different models, may be used to produce a hypothetical solution or to confirm theory ${ }^{[10]}$. One disadvantage of this method is that the rotated factor solution is highly susceptible to the number of factors extracted and may change radically if this is altered. Analytic hierarchy process has the advantage of using the limited information to bring decision for complex problems that are multiple targets, multiple criteria or amorphousness, but the disadvantage of this method is determining weight is subjectivity which may make the result inaccurate.

Taken together, this paper adopts multi-level factor analysis method to establish evaluation model about social organization employees' job satisfaction, the basic idea is: first, we extracted several satisfaction factors from twenty satisfaction indicators, then took factor analysis as criteria level indicators of analytic hierarchy process, the domains of social organization as program level, and compared the social organization employees' job satisfaction in different domains. This method has the following features: (1) it considers the effect of multiple factors on social organization employees' job satisfaction; (2) it can not only understand the employees' job satisfaction in social organization, but also analyses the differences according to the evaluation results of criterion level analysis; (3) compared with the traditional methods about employees' job satisfaction, employees' job satisfaction index can be more comprehensive, and have more powerful and accurate virtues.

\section{Method}

\subsection{Factor Analysis}

Factor analysis is a statistical method that can transfer multiple indicators into a few orthogonal and unobservable random variables through the study of the internal structure of correlation coefficient matrix of original data, and extracts most information of original indicators. When the factor load matrix structure isn't simplified enough, we can rotate factor to make factor more practical significance, at the same time, the factor score function can be presented on the sample evaluation and sorting. The mathematical model of factor analysis is $\underset{(p \times 1)}{X}=\underset{(p \times m)}{A} \underset{(m \times 1)}{F}+\underset{(p \times 1)}{\varepsilon}$, and meet the condition:
(1) $m \leq \rho$;
(2) $\operatorname{Cov}(F, \varepsilon)=0$, it means $F$ and $\varepsilon$ are uncorrelated. 
(3) $D(F)=\left\{\begin{array}{cccc}I & 0 & \ldots & 0 \\ 0 & I & \ldots & 0 \\ \vdots & \vdots & & \vdots \\ 0 & 0 & \ldots & I\end{array}\right\}=I_{m}$, among them, $I=\left\{\begin{array}{cccc}I & 0 & \ldots & 0 \\ 0 & I & \ldots & 0 \\ \vdots & \vdots & & \vdots \\ 0 & 0 & \ldots & I\end{array}\right\}_{p \times p}$ means $F_{1}, \ldots, F_{m}$ are uncorrelated and all variances equal 1 。

$$
D(\varepsilon)=\left\{\begin{array}{cccc}
\delta_{1}^{2} & 0 & \ldots & 0 \\
0 & \delta_{2}^{2} & \ldots & 0 \\
\vdots & \vdots & & \vdots \\
0 & 0 & \ldots & \delta_{p}^{2}
\end{array}\right\} \text { it means } \varepsilon_{1}, \ldots, \varepsilon_{p} \text { are uncorrelated and all variances }
$$

differ each other 。

Among them, $X=\left(X_{1}, \ldots, X_{p}\right)^{\prime}$ is made up of $\mathrm{p}$ observable indicators of $\mathrm{P}$ column random vector; $F=\left(F_{1}, \ldots, F_{m}\right)^{\prime}$ is a unobservable vector, $F$ is common factor of $X$;matrix $A$ is called the factor loading matrix; $a_{i j}$ is known as the factor loading, which is the variable $i$ on the common factor $j ; \varepsilon$ is special factor of $X$, which means the part of variable can't be explained by common factor, all the special factors and common factors are all independent.

\subsection{Analytic Hierarchy Process}

This paper draws multi-level analysis model, which extracts the principal component as measurement indicator of the criterion level, takes different domains in China as a program level, and compares the social organization employees' job satisfaction among different domains. The basic steps of multi-level analysis method are as follows:

1. Establish hierarchical model of the structure, as Figure 1 shows.



Figure 1. Hierarchical Structure Model 
2. The relative importance of element under single criterion

In single level judgment matrix $A$, when $a_{i j}=\frac{a_{i k}}{a_{j k}}$, it is called judgment. The matrix is consistency matrix; we calculate consistency index for judging matrix order; Repeated many times, the mean random consistency index $C I_{i}=\frac{\lambda_{\max }-n}{n-1}$, where $n$ is judgment matrix order; and calculated random consistency indicators $R I ; R I$ is the mathematic average of calculating the random judgment matrix eigenvalues repeatedly. Then we calculate the consistency ratio $C R_{i}, C R_{i}=C I_{i} / R I_{i}$, when $C R_{i}<0.1$, it is generally believed the consistency of judgment matrix is acceptable.

2. Combination weight of element at all levels, as Table 1shows.

Table 1. Combination Weight of Element at all Levels

\begin{tabular}{cccccc}
\hline \multirow{3}{*}{$B$ level } & $A_{1}$ & $A_{2}$ & $\ldots \ldots$ & $A_{m}$ & $\begin{array}{c}\text { Combination } \\
\text { weight of } B \\
\text { level }\end{array}$ \\
\cline { 2 - 6 } & $a_{1}$ & $a_{2}$ & $\cdots \cdots$ & $a_{3}$ & \\
$B_{1}$ & $b_{1}^{1}$ & $b_{1}^{2}$ & $\cdots \cdots$ & $b_{1}^{m}$ & $b_{1}=\sum_{i=1}^{m} a_{i} b_{1}^{i}$ \\
$B_{2}$ & $b_{2}^{1}$ & $b_{2}^{2}$ & $\ldots \ldots$ & $b_{2}^{m}$ & $b_{2}=\sum_{i=1}^{m} a_{i} b_{2}^{i}$ \\
$\vdots$ & $\vdots$ & $\vdots$ & & & $\vdots$ \\
$B_{n}$ & $b_{n}^{1}$ & $b_{n}^{2}$ & $\cdots \cdots$ & $b_{n}^{m}$ & $b_{n}=\sum_{i=1}^{m} a_{i} b_{n}^{i}$ \\
\hline
\end{tabular}

4. The evaluation results of the consistency of total sequencing

Set $C I$ as consistency index of total sequencing, $R I$ as random consistency index of total sequencing, so the formula $C I=\sum_{i=1}^{m} a_{i} C I_{i}, C I_{i}$ is the consistency indicator of judgment matrix of $B$ level corresponding with $A_{i}, R I=\sum_{i=1}^{m} a_{i} R I_{i}, R I_{i}$ is the random consistency indicator of judgment matrix of $B$ level corresponding with $A_{i}$, set $C R=\frac{C I}{R I}$, when $C R \leq 0.10$, we think the results of hierarchy total sequencing have satisfactory consistency.

\section{Empirical Analysis}

\subsection{Sample and Procedure}

From December 2012 to March 2013, we investigated the social organizations of ten cities including the northeast, north, south, southwest and northwest, which can cover the geographical distribution of social organizations in China. We picked up 50 social organizations each city which ranged from the provincial, municipal and district, the areas including the industrial and commercial service industry, agriculture and rural development, scientific research, education, health, culture, sports, ecological environment, social services, legal, and professional 
and professional organization. We distributed a total of 500 questionnaires to social organization staffs, and they filled the blank while the investigators were on-site. Questionnaires with uncompleted answers or suspected unreal answers were excluded. 438 questionnaires with detailed content were collected with the $87.6 \%$ effective rate.

By reference to related literature about the job satisfaction, forming index system, and the requirement of social organization staffs for working conditions in actual investigation, we listed a total of 20 indicators including work burden, the external environment, working environment, freedom of work hours, give full play to the individual in a work ability, accomplishment and self-realization, ease of job development, working interest fit, salary distribution, refresher training, promotion, welfare treatment, rank relationship, relationship with co-worker, the conflict among departments and the concern of leader ${ }^{[1-12]}$. Social organizations from the quality of service evaluation of the actual conditions, we borrowed likert scale, using five steps to the quality of employees' job satisfaction.

\subsection{Reliability and Validity Test}

We used SPSS19.0 and AHP software as the statistical analysis tools. The correlation coefficient matrix is obtained by data processing, the values $p$ are all less than 0.05 , which shows that there are strong correlations among these indicators, we use factor analysis for reducing the dimensionality of complex data sets.

4.2.1. ReliabilityTest: Based on the internal consistency reliability test, the Cronbach's a is 0.952 , higher than 0.8 , which shows the data scale have higher reliability, as Table 2 shows.

Table 2. Reliability Statistics

\begin{tabular}{ccc}
\hline Cronbach's a & $\begin{array}{c}\text { Cronbach's a based on } \\
\text { standardization }\end{array}$ & items \\
\hline 9.937 & 0.952 & 20 \\
\hline
\end{tabular}

4.2.2. Validity Test: With factor analysis, we tested the structural validity of the questionnaires. Factor analysis extracts 4 common factors, and the total cumulative is $60.388 \%$, which reflects the factors are effectively to the scale, as Table 3 showed.

Table 3. Total Variance Explained

\begin{tabular}{|c|c|c|c|c|c|c|}
\hline \multirow{2}{*}{ Element } & \multicolumn{3}{|c|}{ Initial eigenvalue } & \multicolumn{3}{|c|}{ Sum of Square } \\
\hline & total & variance $\%$ & accumulation \% & total & variance $\%$ & accumulation \% \\
\hline 1 & 8.206 & 41.030 & 41.030 & 8.206 & 41.030 & 41.030 \\
\hline 2 & 1.561 & 7.806 & 48.836 & 1.561 & 7.806 & 48.836 \\
\hline 3 & 1.251 & 6.255 & 55.091 & 1.251 & 6.255 & 55.091 \\
\hline 4 & 1.059 & 5.297 & 60.388 & 1.059 & 5.297 & 60.388 \\
\hline 5 & .983 & 4.915 & 65.302 & & & \\
\hline 6 & .811 & 4.053 & 69.356 & & & \\
\hline 7 & .743 & 3.714 & 73.070 & & & \\
\hline 8 & .693 & 3.463 & 76.533 & & & \\
\hline 9 & 647 & 3.235 & 79.768 & & & \\
\hline 10 & .573 & 2.866 & 82.634 & & & \\
\hline 11 & .498 & 2.488 & 85.122 & & & \\
\hline 12 & .485 & 2.427 & 87.550 & & & \\
\hline 13 & .464 & 2.321 & 89.870 & & & \\
\hline 14 & .444 & 2.218 & 92.089 & & & \\
\hline 15 & .355 & 1.776 & 93.865 & & & \\
\hline 16 & .309 & 1.545 & 95.410 & & & \\
\hline 17 & .303 & 1.516 & 96.927 & & & \\
\hline 18 & .242 & 1.210 & 98.136 & & & \\
\hline 19 & .215 & 1.074 & 99.210 & & & \\
\hline 20 & .158 & .790 & 100.000 & & & \\
\hline
\end{tabular}


Table 3. Total Variance Explained

\begin{tabular}{|c|c|c|c|c|c|c|}
\hline \multirow{2}{*}{ Element } & \multicolumn{3}{|c|}{ Initial eigenvalue } & \multicolumn{3}{|c|}{ Sum of Square } \\
\hline & total & variance $\%$ & accumulation \% & total & variance $\%$ & accumulation \% \\
\hline 1 & 8.206 & 41.030 & 41.030 & 8.206 & 41.030 & 41.030 \\
\hline 2 & 1.561 & 7.806 & 48.836 & 1.561 & 7.806 & 48.836 \\
\hline 3 & 1.251 & 6.255 & 55.091 & 1.251 & 6.255 & 55.091 \\
\hline 4 & 1.059 & 5.297 & 60.388 & 1.059 & 5.297 & 60.388 \\
\hline 5 & .983 & 4.915 & 65.302 & & & \\
\hline 6 & .811 & 4.053 & 69.356 & & & \\
\hline 7 & .743 & 3.714 & 73.070 & & & \\
\hline 8 & .693 & 3.463 & 76.533 & & & \\
\hline 9 & .647 & 3.235 & 79.768 & & & \\
\hline 10 & .573 & 2.866 & 82.634 & & & \\
\hline 11 & .498 & 2.488 & 85.122 & & & \\
\hline 12 & .485 & 2.427 & 87.550 & & & \\
\hline 13 & .464 & 2.321 & 89.870 & & & \\
\hline 14 & .444 & 2.218 & 92.089 & & & \\
\hline 15 & .355 & 1.776 & 93.865 & & & \\
\hline 16 & .309 & 1.545 & 95.410 & & & \\
\hline 17 & .303 & 1.516 & 96.927 & & & \\
\hline 18 & .242 & 1.210 & 98.136 & & & \\
\hline 19 & .215 & 1.074 & 99.210 & & & \\
\hline 20 & .158 & .790 & 100.000 & & & \\
\hline
\end{tabular}

\subsection{Analysis}

4.3.1. Factor Analysis: The rotation matrix in Table 4 shows: decision level, relationship with co-workers, the concern of leader to subordinate, recognition to corporate culture, job stability, job interest, work autonomy, rules and regulations are high correlated with the first common factor; welfare treatment, work burden, work environment, educational training and salary distribution are high correlated with the second common factor; job challenge, performance show, job meaning are high correlated with the third common factor; Job promotion, ability promotion, leader and co-worker approval, communication opportunity are high correlated with the fourth common factor. We named the four common factors as staff cognition, work rewards, work value and room for development.

Table 4. Rotation Matrix

\begin{tabular}{|l|r|r|r|r|}
\hline & \multicolumn{4}{|c|}{ Elements } \\
\cline { 2 - 5 } & 1 & 2 & 3 & \multicolumn{1}{|c|}{} \\
\hline Decision-making level & .722 & .268 & .176 & .218 \\
Relationship with co-workers & .713 & -.040 & .009 & .297 \\
The concern of leader to subordinate & .674 & .378 & .224 & .187 \\
Recognition to corporate culture & .639 & .123 & .289 & .174 \\
Job stability & .629 & .393 & .218 & .126 \\
Job interest & .556 & .149 & .385 & .336 \\
Work autonomy & .503 & .408 & .166 & .341 \\
Rules and regulations & .466 & .463 & .206 & .368 \\
Welfare treatment & .207 & .694 & .376 & .325 \\
Work burden & .058 & .669 & -.232 & .097 \\
Work environment & .459 & .611 & -.066 & -.084 \\
Educational training & .109 & .589 & .431 & .123 \\
Salary distribution & .237 & .559 & .512 & .168 \\
Job challenge & .201 & -.063 & .766 & .145 \\
Performance show & .105 & .121 & .687 & .145 \\
Job meaning & .504 & .036 & .594 & -.058 \\
Job promotion & .230 & .080 & -.137 & .737 \\
Ability promotion & .120 & .087 & .313 & .686 \\
Leader and co-worker approval & .357 & .220 & .368 & .542 \\
Communication opportunity & .350 & .262 & .275 & .491 \\
\hline
\end{tabular}


4.3.2. Multi-level Analysis: We draws factor analysis method to reduce the dimensionality of 20 factors about social organization employees' satisfaction, and get four principal components, namely staff cognition, work rewards, work value and room for development as the criterion level, social organization employees' job satisfaction from ten fields as program level, target level is the social organization employees' job satisfaction. The result of AHP software showed as follows:

$A \rightarrow C$ :the maximum eigenvalue $\lambda$ max $=4.726$, the corresponding eigenvectors are [0.182, $0.021,0.214,0.018] T, C I=0.019, R I=1.120, C R=0.03, C R<0.1$, consistency test passes; the sorts of four principal component's weight as follows: staff cognition, work value, room for development, work reward.

$B \rightarrow C_{1}$ : the maximum eigenvalue $\lambda \max =10.000$, the corresponding eigenvectors are $[0.097$, $0.106,0.107,0.098,0.106,0.100,0.097,0.096,0.102,0.091] T, C I=0.000, R I=1.490, C R=0.000$, $C R<0.1$, consistency test passes; the sorts of staff cognition satisfaction as follows: health, labor, culture, education, technology, civil affairs, others, sports, social medium service and legal service.

$B \rightarrow C_{2}$ : the maximum eigenvalue $\lambda \max =10.000$, the corresponding eigenvectors are $[0.101,0.105,0.103,0.102,0.107,0.099,0.099,0.094,0.104,0.092] T, C I=0.000, R I=1.490, C R$ $=0.000, C R<0.1$, consistency test passes; the sorts of work environment satisfaction as follows: technology, education, health, culture, legal service, civil affairs, labor, sports, social medium service and others.

$B \rightarrow C_{3}$ : the maximum eigenvalue $\lambda \max =10.000$, the corresponding eigenvectors are $[0.096,0.101,0.104,0.095,0.096,0.099,0.092,0.090,0.099,0.103] T, C I=0.000, R I=1.490, C R$ $=0.000, C R<0.1$, consistency test passes; the sorts of room for development satisfaction as follows: culture, health, education, legal service, labor, technology, sports, social medium service, civil affairs and others.

$B \rightarrow C_{4}$ : the maximum eigenvalue $\lambda \max =10.000$, the corresponding eigenvectors are $[0.099,0.101,0.096,0.099,0.107,0.103,0.096,0.102,0.096,0.096] T, C I=0.000, R I=1.490, C R$ $=0.000, C R<0.1$,consistency test passes; the sorts of work rewards satisfaction as follows: health, education, technology, culture, labor, legal service, social medium service, civil affairs, others and sports.

Hierarchical total sequencing: $C I=0.000, R I=1.490, C R=0.000, C R<0.1$, consistency test passes; the weight vector $\mathrm{W}=[0.099,0.105,0.101,0.098,0.099,0.097,0.107,0.099$, $0.103,0.099] T$, it means health, education, labor, technology, culture, legal service, social medium service, civil affairs, others and sports.

\section{Findings}

\subsection{Social Organization Staff's Job Satisfaction Scores Lower}

According to the analysis of survey data, the comprehensive level of social organization employees' job satisfaction is 2.06. Among them, the score of staff cognition is 3.29, the score of work value is 2.49 , the score of room for development is 3.37, and the score of work rewards is 2.96. Overall, social organization employees work in a stressful and uncertain environment, facing multiple pressures on financial, political, and managerial, so their job satisfaction stays in low levels, but the work environment and work rewards are in general level, which means we should take effective measures to improve job environment and provides the feasible future career planning, and broaden the personal room for development, then enhance the level of satisfaction.

\subsection{Analytic Hierarchy Process of $A \rightarrow C$, the Weight of Staff Cognition is Greatest}

The data from the first hierarchy analysis of $A \rightarrow C_{\text {shows: the weight of staff cognition is }}$ greatest in all the four criteria, work value ranks second, and then is room for development and 
employment rewards. Through the principal component elements extracted by factor analysis: the first principal components is staff cognition which includes decision level, relationship with co-workers, the concern of leader to subordinate, recognition to corporate culture, job stability, job interest, work autonomy, rules and regulations. From the content, it mainly includes the supervisor relations, working nature and working atmosphere, the staff cognition scores higher than room for development and work rewards because of the characteristics, thinking pattern and working nature in social organization, which shows that human resources management of social organization is different with for-profit organizations ${ }^{[13]}$. People choose to enter social organization mainly because they have the high moral sentiment and dedication, they like open and free working environment, they pay more attention to effective communication, self-worth and fair treatment than the promotion or welfare treatment etc. If social organization can't provide such working environment for staffs, they may cause cognitive dissonance, drop trust and loyalty to the whole industry and turnover intention is at a high level.

\subsection{Analytic Hierarchy Process of $B \rightarrow C_{1} C_{2} C_{3} C_{4}$ - Regional Comparison}

Through analytic hierarchy process of $B \rightarrow C_{1} 、 B \rightarrow C_{2} 、 B \rightarrow C_{3} 、 B \rightarrow C_{4}$, we can get the sort of staff cognition, work value, room for development, employment rewards in different domains. From which we can see employment rewards and human relations of health score highest, room for development and work value of education score highest, while sports reports the lowest scores all over the domains. We suggest that the relevant social organizations or authorities in sports should pay attention to this phenomenon, draw lessons from the cities that get relatively higher scores, enhance the social organization employees' job satisfaction as soon as possible. In addition, others and sports should take employment reward and room for development on diagnosis, put forwards corresponding policies to promote the social organization employees' job satisfaction.

\subsection{Hierarchical Total Sequencing - Comprehensive Regional Comparison}

Based on the final weight vector through multi-level analysis, we get the hierarchical total sequencing: health, education, labor, technology, culture, legal service, social medium service, civil affairs, others and sports. The top three relatively optimal cities overall comprehensive levels are health, education and labor, the two relatively worst are others and sports. The final sequencing not only reflects the regional level of social organization employees' satisfaction, but also reveals the people who worked in these domains have weaker awareness of charity than the other domains. So the competent authorities throughout China should put in place policies and enhance the level of social organization employees' job satisfaction according to research conclusion of the paper.

\section{Conclusion}

In this paper, we evaluated and compared the social organization employee's job satisfaction through the method of the factor analysis and multi-level analysis. On the one hand, we considered the influence of multiple factors on social organization employees' job satisfaction; On the other hand, this method can not only understand the job satisfaction of social organization, but also finds out the problems in the human resource management of social organizations according to the result of criterion level's evaluation. Social organization employees' job satisfaction is an important symbol of social organization maturity, we often carries on the observation and analysis, can help the social organization to understand their own development condition, and take steps to increase the attraction to human resource of the organization. Our research indicates the social organization should advocate organization culture and set organizational goals to enhance group cohesion, use organizational behavior to stimulate and restrain individual behavior, and call up the individual members the sense of responsibility and mission, and the identity of the masses to make it feel their value. On the other hand, social organization should put forward to the objectives and 
principles to guide, persuade, and encourage staffs that can stimulate their intrinsic motivation, instead of keening on regulations, structure and mode. At the same time, it can provide reference and basis for the competent authorities to get information of social organization employees' job satisfaction in time, and implement macroeconomic management policies or strategies.

\section{Acknowledgements}

A first draft of this paper was presented at the $3^{\text {rd }}$ international seminar on Philanthropy in December 2013. Many thanks to Prof. Chen. X.Q, (MUC) who was co-convener of the session, as well as to the other speakers and audience, who gave valuable feedback on the paper. The author also acknowledge financial support from the national social science fund major project - the empirical study on ngos involving in the ethnic culture development(13AMZ003).

\section{References}

[1]. C. Ellenbecker, "Home Health Care Nurses' job satisfaction: a System Indicatior.", Home Health Care Management and Practice, vol. 6, no. 13, (2001)

[2]. R. Caers, C. D. Bois, M. Jegers, S. D. Gieter, R .D. Cooman and R. Pepermans, 'Measuring Community nurses' job satisfaction: literature Review", Journal of Advanced Nursing, vol. 5, no. 62, (2008).

[3]. C. Hale, "Measuring Job Satisfaction Nursing Times", vol. 5, no. 82, (1986).

[4]. W. Pilkington and J. Wood, "Job Satisfaction, Role Conflict and Role Ambiguity a Study of Hospital Nurses", Australian Journal of Advanced Nursing, vol. 3, no. 3, (1986).

[5]. M. A. Blegen and C. W. Muller, "Nurses' Job Satisfaction: a Longitudinal Analysis", Research in Nursing and Health, vol. 4, no. 10, (1987)

[6]. J. Bush, "Job Satisfaction, Powerlessness and Locus of Control", Western Journal of Nursing Research, vol. 6, no. $10,(\mathbf{1 9 8 8})$.

[7]. D. L. Salamon, "Partners in Public Service: Government-Nonprofit Relations in the Modern Welfare State", The Johns Hopkins University Press, Baltimore, (1995).

[8]. W. A. Chess, J. M. Norlin and S. D. Jayaratne, "Social Work Administration 1981-1985: Alive, Happy and Prospering. Administration in social Work", vol. 2, no. 11, (1987).

[9]. E. Hamarta, "A Prediction of Self-esteem and Life Satisfaction by Social Problem Solving", Social Behavior and Personality, vol. 1, no. 37, (2009).

[10]. B. G. Tabachruck and L. S. Fidell, “Using Multivariate Statistics”, Harper and Row, New York, (1983).

[11]. W. Traynor, "The Development of A Measure of Job Satisfaction for Use in Monitoring the Morale of Community Nurses in Four Trusts", Journal of Advanced Nursing, vol. 1, no. 18, (1993).

[12]. N. J. Jimmieson and M. A. Griffin, "Linking Client and Employee Perceptions of the Organization: A Study of s71, (1998).

[13]. C. Mclaughlin, “The Management of Nonprofit Organization”, John Wiley and Sons, New Jersey, (1986). 
International Journal of $u-$ and e- Service, Science and Technology Vol.8, No.2 (2015) 